\title{
Tumor-specific hyperthermia with aptamer-tagged superparamagnetic nanoparticles
}

\author{
This article was published in the following Dove Press journal: \\ International Journal of Nanomedicine \\ 17 December 2013 \\ Number of times this article has been viewed
}

\author{
Katarzyna Pala ${ }^{1,2}$ \\ Anna Serwotka' \\ Filip Jeleń \\ Piotr Jakimowicz' \\ Jacek Otlewski ${ }^{1,2}$ \\ 'Department of Protein Engineering, \\ Faculty of Biotechnology, University \\ of Wroclaw, Wroclaw, Poland; \\ ${ }^{2}$ Wroclaw Research Centre EIT+, \\ Wroclaw, Poland
}

\begin{abstract}
Targeted therapy is a method owing to its limited side effect profile, particularly in cancer treatment. Magnetic hyperthermia, which is induced by nanoparticles (NPs) conjugated with targeting agents, can be useful in combination with chemo- or radiotherapy. In this paper, we constructed dextran-coated ferric oxide NPs conjugated with specific anti-human epidermal growth factor receptor (HER2) aptamer and used them to induce magnetic hyperthermia in cultured cells. The specificity of the tagged NPs was determined by studying their effect relative to that of non-tagged NPs against two cell lines: human adenocarcinoma SK-BR3, overexpressing the HER2 receptor; and U-87 MG, a human glioblastoma epithelial cell line, not expressing HER2. In order to confirm the interaction of the tagged NPs with the cells we used, fluorescence microscopy and fluorescence-activated cell sorting analysis were performed. All of these experiments showed that the aptamer-tagged NPs were highly specific toward the HER2-expressing cells. In addition, a ninetyfold lower dose of the tagged NPs relative to that of the non-tagged NPs was needed to achieve $\sim 50 \%$ cell killing by hyperthermia of the SK-BR3 cell line, while for the U-87 MG cells the viability level was close to $100 \%$. These results show that targeted NPs can be applied at substantially lower doses than non-targeted ones to achieve similar effects of hyperthermia, which should greatly limit the side effects of treatment.
\end{abstract}

Keywords: superparamagnetic nanoparticles, hyperthermia, aptamer, targeted therapy

\section{Introduction}

During the last 2 decades, nanotechnology has entered new areas of application, including biotechnology and pharmacology. The diversity of nanoparticles (NPs) has allowed for the development of innovative technologies in drug formulation, targeted therapy, drug delivery, and diagnostics. Nanomaterials such as nanorods, nanospheres, or NPs display unique physical, optical, and chemical properties ${ }^{1-3}$ which, in combination with their large surface-to-volume ratio, make them useful for cancer diagnosis and treatment. Numerous approaches to manufacture functional nanomaterials for anticancer therapy have been proposed and using nanoparticles as vehicles which carry both a targeting component and a specific drug one can improve the delivery of diverse agents into cells and tissues. ${ }^{4}$

Magnetic NPs attract strong interest due to their associated hyperthermic effects and their ease of conjugation with biological components. A unique physical phenomenon called superparamagnetism causes an increase of their temperature when subjected to an alternating magnetic field. ${ }^{5}$ The effect offers novel approaches to cancer treatment. For example, the therapy evaluated by Jordan et al ${ }^{6,7}$ relies on the injection of NPs directly into solid tumors, which are then selectively heated by an externally applied
Correspondence: Jacek Otlewski Department of Protein Engineering, Faculty of Biotechnology, University of Wroclaw, F. Joliot-Curie 14a, 50-383

Wroclaw, Poland

Tel +48 7I 3752824

Fax +48 7I 3752608

Email otlewski@protein.pl
International Journal of Nanomedicine 2014:9 67-76 (c) (i) (5) 2014 Pala et al. This work is published by Dove Medical Press Limited, and licensed under Creative Commons Attribution - Non Commercial (unported, v3.0) License. The full terms of the License are available at http://creativecommons.org/licenses/by-nc/3.0/. Non-commercial uses of the work are permitted without any further permisson fove Medical Press Limited, provided the work is properly attributed. Permissions beyond the scope of the License are administered by Dove Medical Press Limited. Information on how to request permission may be found at: http://www.dovepress.com/permissions.php 
magnetic field. The rise of temperature induces the cancer cells to undergo apoptosis or necrosis and makes the tumor more vulnerable to radio- or chemotherapy. While hyperthermia alone is often not sufficient when treating cancer, it greatly improves other conventional modalities like radio-, chemo-, or immunotherapy. ${ }^{8-10}$

Modern anticancer therapies are based on the targeted delivery of active substances into the tumor cells, which reduces harmful effects in healthy tissues, allowing specific tumor destruction. Recently, aptamers are becoming an increasingly popular class of targeting agents, and they offer an interesting alternative to antibodies. These oligonucleotides are capable of forming three-dimensional structures and interact strongly and selectively with macromolecular targets. The use of aptamers as targeting agents has several advantages. Compared to antibodies, aptamers are much more stable across a broad range of $\mathrm{pH}$ values, organic solvents, and temperatures. Aptamers are easily modified by fluorophores, NPs, toxic agents, and so on, without decreasing their binding capabilities. ${ }^{11,12}$ These features make aptamers suitable for many diagnostic and therapeutic applications. Conjugated with gold NPs (AuNPs) ${ }^{13}$ they act as sensitive diagnostic agents mainly in fast colorimetric assays. A combination of aptamers and magnetic NPs can be used for the detection of small molecules and proteins. ${ }^{14}$ Cao et $\mathrm{al}^{15}$ demonstrated the use of liposomes conjugated with an aptamer for the effective delivery of cisplatin to cancer cells.

In the present work, we designed multifunctional magnetic NPs conjugated with a specific anti-human epidermal growth factor receptor (HER) 2 aptamer and used them for targeted hyperthermia. Our results show that targeted hyperthermia is more effective in killing cancer cells than non-targeted magnetic NPs.

\section{Materials and methods Synthesis of dextran-coated magnetic nanoparticles}

We used carboxylated dextran to obtain high-density functionalized groups of magnetic NPs. Ten gram of dextran (S10; Pharmacia, Stockholm, Sweden) was dissolved in $100 \mathrm{~mL}$ of $1 \mathrm{M}$ bromoacetic acid in $2 \mathrm{M}$ sodium hydroxide, and it was mixed overnight at room temperature (RT). ${ }^{16}$ The solution was then dialyzed against Milli-Q water (EMD Millipore, Billerica, MA, USA) and used for nanoparticle synthesis.

The preparation of magnetic NPs coated with carboxylated dextran was performed according to Hradil et al. ${ }^{17}$ The aqueous solution of carboxylated dextran was mixed with
$1.51 \mathrm{~g}$ of $\mathrm{FeCl}_{3} \cdot 6 \mathrm{H}_{2} \mathrm{O}$ and $0.64 \mathrm{~g}$ of $\mathrm{FeCl}_{2} \cdot 4 \mathrm{H}_{2} \mathrm{O}$. Twenty microliters of $7.5 \% \mathrm{NH}_{4} \mathrm{OH}$ solution was added, causing black precipitate formation during 30 minutes of incubation at $60^{\circ} \mathrm{C}$. The precipitate was washed several times with Milli-Q water until the $\mathrm{pH}$ was close to neutral.

The size distribution of NPs was evaluated by dynamic light scattering (DynaPro NanoStar ${ }^{\mathrm{TM}}$; Wyatt Technology, Santa Barbara, CA, USA) and transmission electron microscopy (TEM) (FEI Tecnai ${ }^{\mathrm{TM}}$ G2 20 X-TWIN; FEI, Hillsboro, OR, USA). The crystal structure was examined by X-ray diffraction (XRD) using a D8 ADVANCE powder diffractometer with $\mathrm{Cu} \mathrm{K} \alpha$ radiation and a Vantec detector (Bruker Optik GmbH, Ettlingen, Germany).

The superparamagnetic properties of NPs were measured by superconducting quantum interference device (SQUID) analysis (Quantum Design, Inc, San Diego, CA, USA) and confirmed by the rise of the temperature under the magnetic field analysis.

\section{Conjugation reaction of anti-Her2 aptamer with dextran-coated nanoparticles}

Modified anti-HER2 aptamer with 5' thiol group attached (molecular weight $=14,360.1 \mathrm{Da}$ ) was purchased from Aptamer Sciences Inc (Pohang, South Korea) (\#SH-1194-35). The aptamer contained benzyl, napthyl, or tryphtophyl nucleosides, which were incorporated to increase affinity (Kd [dissociation constant] of $13 \mathrm{nM}$ toward the extracellular domain of HER2) and serum half-life ( 70 hours versus 3 hours for unmodified deoxyribonucleic acid [DNA] aptamers). The aptamer was dissolved in systematic evolution of ligands by exponential enrichment (SELEX) buffer ( $40 \mathrm{mM}$ of 2-[4-\{2-hydroxyethyl $\}$ piperazin-1-yl] [HEPES], pH 7.5; $100 \mathrm{mM}$ of NaCl; $5 \mathrm{mM}$ of $\mathrm{KCl}$; and $5 \mathrm{mM}$ of $\mathrm{MgCl}_{2}$ ) to the final concentration of $1 \mu \mathrm{g} / \mu \mathrm{L}$.

In order to obtain NPs coupled with a fluorescent probe, which could be suitable for a flow cytometry analysis, conjugation of the aptamer to the NPs was carried out in two steps. First, the NPs were dispersed in $50 \mathrm{mM}$ of 2-(N-morpholino)ethanesulfonic acid buffer ( $\mathrm{pH} 5.5$ ), and the carboxylic groups were activated with carbodiimide 1-ethyl-3-(3-dimethylaminopropyl) carbodiimide (EDC) and sulfo-N-hydroxysulfosucciniide (NHS) (Thermo Fisher Scientific, Waltham, MA, USA) and then modified with the heterobifunctional linker 3-(2-pyridyldithio)propionyl hydrazide (PDPH) (Thermo Fisher Scientific) and 6-aminofluorescein (AF) (Sigma-Aldrich, St Louis, MO, USA). Two milligrams of EDC and sulfo-NHS was added to $1 \mathrm{~mL}$ of NP solution $(1 \mathrm{mg} / \mathrm{mL})$. Concurrently, PDPH and AF 
were added to a final concentration of $2.2 \mathrm{mM}(\mathrm{PDPH})$ and $0.44 \mathrm{mM}$ (AF), respectively. After 2 hours of incubation at RT, the reaction was washed three times with SELEX buffer and the material reduced with $1 \mathrm{mM}$ tris(2-carboxyethyl)phosphine (Thermo Fisher Scientific), followed by triple washes. In the interim, the solution of aptamer $(0.5 \mathrm{~mL} 1.4 \mu \mathrm{M}$ in Systematic Evolution of Ligands by Exponential Enrichment (SELEX) buffer; $\mathrm{pH}$ 7.5) was boiled for 5 minutes, cooled to RT, and added to the prepared NP solution. The mixture was incubated overnight at RT followed by triple washing with SELEX buffer.

Hereafter, we will use the abbreviation, NPAF (nanoparticleaptamer-fluorescein) to denote the dextran-coated, aptamerbound, AF-labeled magnetic NPs obtained as above. In the case of the hyperthermia experiment, the NPs without fluorescent probe were synthesized according to the same procedure.

The presence of the aptamer molecules on the surface of the NPs was confirmed and quantified by a fluorescence assay with the Quant-iT ${ }^{\mathrm{TM}}$ OliGreen ${ }^{\circledR}$ ssDNA Reagent and Kit (Life Technologies, Carlsbad, CA, USA), which is suitable for the quantitative measurement of single-stranded DNA.

\section{Cell cultures}

The experiments were performed on a human adenocarcinoma SK-BR3 cell line. The cells were maintained in McCoy's 5A medium supplemented with $10 \%$ fetal bovine serum, $3 \mathrm{mM}$ of L-glutamine, and antibiotics McCoy's 5A medium supplemented with $10 \%$ fetal bovine serum, $3 \mathrm{mM}$ of L-glutamine, and antibiotics (Cytogen $\mathrm{GmbH}$, Sinn, Germany). As a control for all of the experiments, the human glioblastoma epithelial cell line U-87 MG was used. The U-87 MG cells were maintained in minimum essential medium supplemented with $10 \%$ fetal bovine serum, $4 \mathrm{mM}$ L-glutamine, and antibiotics.

\section{Flow cytometry measurement}

The specificity of NPs coated with the anti-HER2 aptamer was investigated by fluorescence activated cell sorting (FACS) (FACSCalibur ${ }^{\mathrm{TM}}$; BD Biosciences, San Jose, CA, USA). Cells were seeded onto a Petri dish in complete medium and grown to $80 \%$ confluence. NPs modified with the anti-HER2 aptamer and AF (NPAF) were added to each plate and incubated for 15 minutes at $37^{\circ} \mathrm{C}, 5 \% \mathrm{CO}_{2}$. Before the measurements were conducted, the samples were washed three times with phosphate buffered saline (PBS), followed by trypsinization and one more washing with $\mathrm{PBS}(\mathrm{pH}=7.5)$.

\section{Fluorescence imaging measurement}

To confirm the interactions of the targeted NPs, we also performed a fluorescence microscopy experiment (Observer Z1;
Carl Zeiss Meditec AG, Jena, Germany). We used the AF-labeled aptamer-NP conjugates (NPAF). For the control, we prepared the aptamer modified with AF and herceptin conjugated with fluorescein isothiocyanate (FITC) herceptin conjugated with FITC (HF). The U-87 MG cell line was used as a negative control.

SK-BR3 and U-87 MG cells were cultured on glass coverslips in full medium to $80 \%$ confluence. Cells were washed with PBS and fixed with 4\% paraformaldehyde (15 minutes at RT) followed by triple washes with PBS buffer and blocking with 5\% bovine serum albumin in PBS for 15 minutes at RT. Appropriate compounds (NPs conjugated with AF or NPAF; aptamer conjugated with $\mathrm{AF}$; or herceptin conjugated with FITC or HF) were incubated with cells at $37^{\circ} \mathrm{C}$ for $15 \mathrm{~min}-$ utes. Finally, the coverslips were washed three times with PBS buffer and mounted in VECTASHIELD ${ }^{\circledR}$ Hard Set Mounting Medium with 4',6-diamidino-2-phenylindole (DAPI) (Vector Laboratories, Inc, Burlingame, CA, USA). The slides were visualized under the fluorescence microscope.

\section{Hyperthermia caused by targeted and non-targeted nanoparticles}

SK-BR3 and U-87 MG cells were grown on Petri plates to $80 \%$ confluence in full medium. On each cell line, two groups of experiments were performed to check the effects of ordinary non-targeted iron oxide NPs and targeted NPs. In the first group, four variants were run: the sample with NPs and the magnetic field $(\mathrm{CN}+)$; the control samples with NPs without the magnetic field $(\mathrm{CN}-)$; the magnetic field without NPs $(\mathrm{C}+)$; and control cells without NPs or the magnetic field (C-). Dextran-coated iron oxide colloid solution (1 mL) was added to the $\mathrm{CN}+$ and $\mathrm{CN}$ - plates to a final concentration of $1 \mathrm{mg} / \mathrm{mL}$. After 30 minutes of incubation at $37^{\circ} \mathrm{C}, 5 \% \mathrm{CO}_{2}$ cells were washed with medium to remove unbound NPs. The magnetic field (300A, 280 kHz; Easyheat; Ambrell, Scottsville, $\mathrm{NY}$, USA) was applied to the $\mathrm{CN}+$ and $\mathrm{C}+$ plates twice for 30 minutes at 30-minute intervals. The viability of cells was evaluated after overnight incubation at $37^{\circ} \mathrm{C}, 5 \% \mathrm{CO}_{2}$ by the Trypan blue (Life Technologies) staining method.

In the second group, six variants were run. All of them are described in Table 1. SK-BR3 and U-87 MG cell lines were treated with aptamer-modified NPs at a final concentration of $11 \mu \mathrm{g} / \mathrm{mL}$. The control samples were treated with NPs without aptamer molecules and with a free, unconjugated anti-HER2 aptamer $(0.1 \mu \mathrm{g} / \mathrm{mL})$. The magnetic field (300 A, $280 \mathrm{kHz})$ was applied twice for 30 minutes at a 30 -minute interval. After overnight incubation at $37^{\circ} \mathrm{C}, 5 \% \mathrm{CO}_{2}$, the viability of the cells was evaluated by the Trypan blue staining method. The statistical analysis was performed using Student's $t$-test. 
Table I Summary of experimental variants in targeted hyperthermia

\begin{tabular}{ll}
\hline Abbreviation & Cell treatment \\
\hline CNA+ & Aptamer-modified nanoparticles and a magnetic field \\
CNA- & $\begin{array}{l}\text { Aptamer-modified nanoparticles without a magnetic } \\
\text { field }\end{array}$ \\
$\mathrm{CN}^{+}$ & Dextran-coated nanoparticles and a magnetic field \\
$\mathrm{CN}-$ & Dextran-coated nanoparticles without a magnetic field \\
$\mathrm{CA}+$ & Aptamer and a magnetic field \\
$\mathrm{CA}-$ & Aptamer without a magnetic field \\
$\mathrm{C}_{+}$ & Magnetic field \\
$\mathrm{C}_{-}$ & Control cells \\
\hline
\end{tabular}

\section{Results}

\section{Synthesis and characterization} of nanoparticles

We prepared magnetic NPs coated with dextran since this natural polymer is biocompatible and bears functional groups that are easy to modify. The size and monodispersity of the NPs were determined by dynamic light scattering (Figure 1). NPs were dispersed in Milli-Q water at a concentration of $0.01 \mathrm{mg} / \mathrm{mL}$, and the experiment was performed at $25^{\circ} \mathrm{C}$. The average hydrodynamic diameter of the NPs was slightly below $70 \mathrm{~nm}$, and the solution did not show any aggregates.

Figure 2 shows the TEM image (Figure 2A) and the XRD pattern (Figure 2B) of iron oxide NPs. The TEM analysis showed uniform distribution of the NPs, and their diameter was about $10 \mathrm{~nm}$. The XRD patterns (Figure 2B) revealed peaks that were characteristic of $\Upsilon-\mathrm{Fe}_{2} \mathrm{O}_{3}$.

The superparamagnetic properties of iron oxide NPs were displayed by SQUID analysis, which was performed at RT
(Figure S1). Magnetization of the sample is almost temperatureindependent, and its values rise fast with field increases.

In order to confirm the ability to increase the temperature under the magnetic field, the temperature of the water solution with different concentrations of magnetic NPs was measured. The results showed that the higher the NP concentration, the faster the temperature increases, which is a consequence of the superparamagnetic properties of the NPs (Figure S2).

\section{Conjugation of nanoparticles with aptamer anti-Her2}

The anti-HER2 aptamer is a commercially available molecule that is modified with a thiol group. The aptamer was conjugated with dextran-coated NPs through a commercially available linker (PDPH) to introduce the proper functional group onto the NP surface. The same linker was also used to incorporate a fluorescent probe for use in the $\mathrm{NP}$-cell interaction analysis. After the conjugation, the NPs were sonicated for 30 minutes just before the analysis or further experiments were conducted.

The final concentration of the aptamer, measured by the Quant-iT ${ }^{\mathrm{TM}}$ OliGreen ${ }^{\circledR}$ ssDNA Reagent and Kit, was $5 \mu \mathrm{g} / \mathrm{mL}$.

\section{Interaction of aptamer-conjugated nanoparticles with HER2-overexpressing cells}

The specificity of the aptamer-modified NPs was analyzed by FACS analysis and fluorescence microscopy using aptamer-conjugated NPs that were modified with AF. The

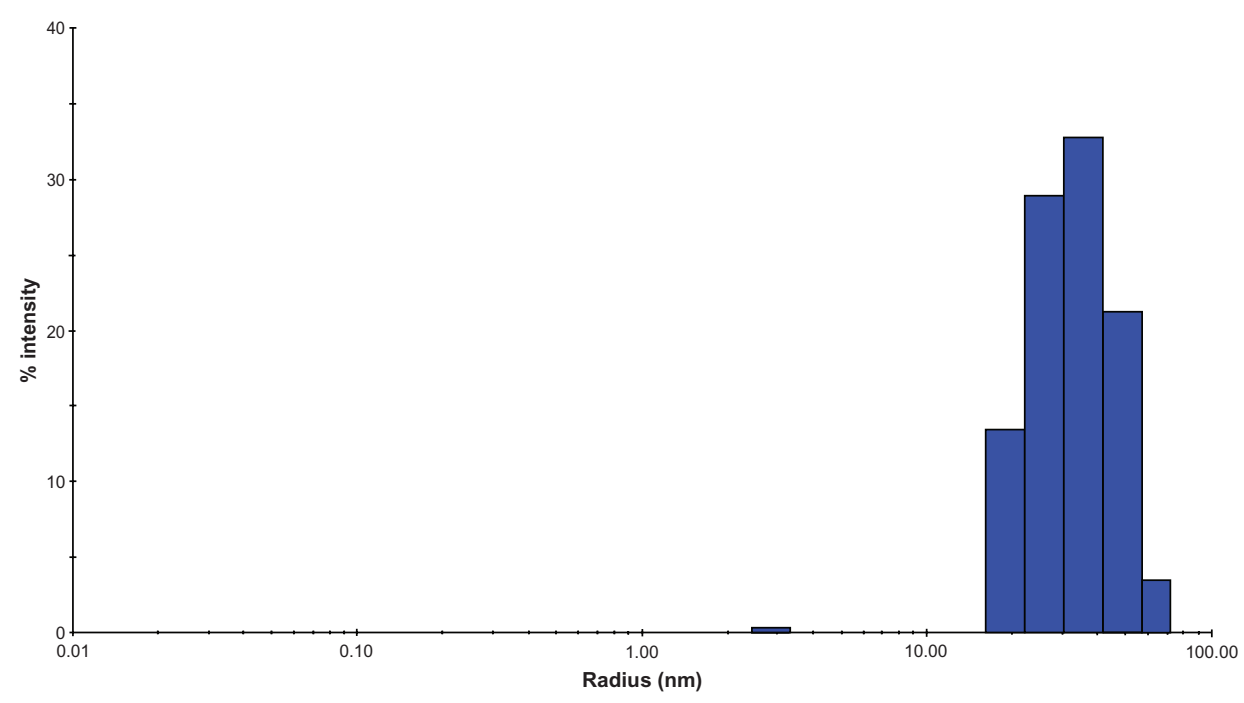

Figure I Dynamic light scattering of dextran-coated magnetic nanoparticles.

Notes: Dynamic light scattering measured in $\mathrm{H}_{2} \mathrm{O}$ Milli-Q (EMD Millipore, Billerica, MA, USA) at $25^{\circ} \mathrm{C}$. 

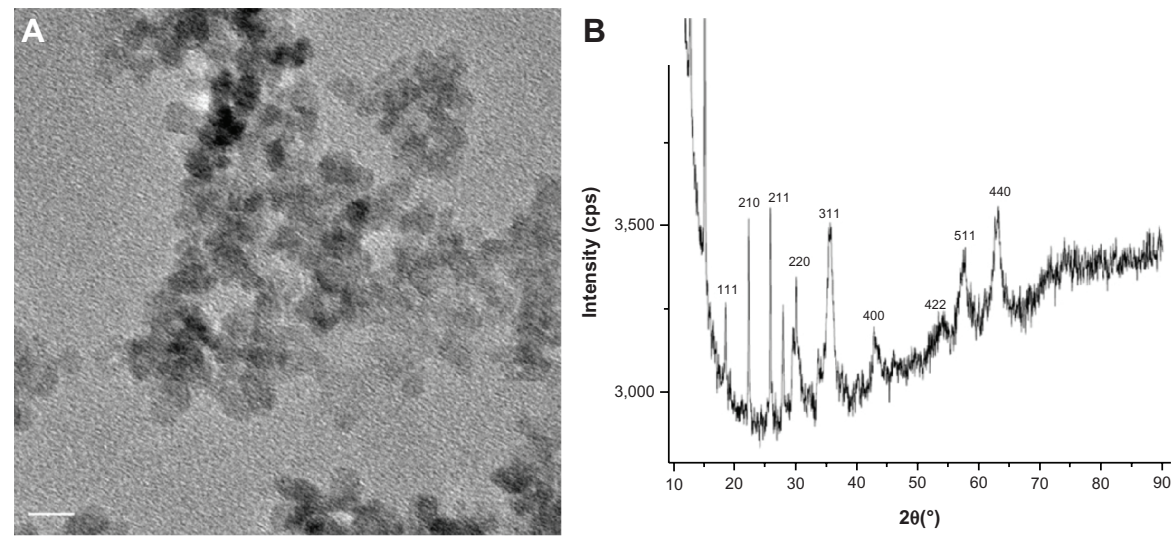

Figure 2 Transmission electron microscopy image and X-ray diffraction analysis of iron oxide nanoparticles coated with dextran.

Notes: (A) Transmission electron microscopy image and (B) X-ray diffraction analysis of iron oxide nanoparticles coated with dextran. The white bar corresponds to $10 \mathrm{~nm}$.

U-87 MG cell line was used as a negative control, and HF was used as a positive control.

The FACS histograms show that NPAF could be efficiently bound by the HER2-expressing SK-BR3 cells (Figure 3A), while the HER2-negative U-87 MG cells were not (Figure 3B). This result showed that the NPs were indeed modified with a properly-folded and active aptamer that provided specific functionality.

The fluorescence imaging experiment confirmed a specific interaction between the aptamer-modified NPs with cancer cells that overexpress HER2 receptors (Figure 4). In the case of the SK-BR3 cell line, we observed a strong green fluorescence of cells treated with the targeted NPs conjugated with AF, which is in clear contrast to the U-87 MG cells.

\section{A}

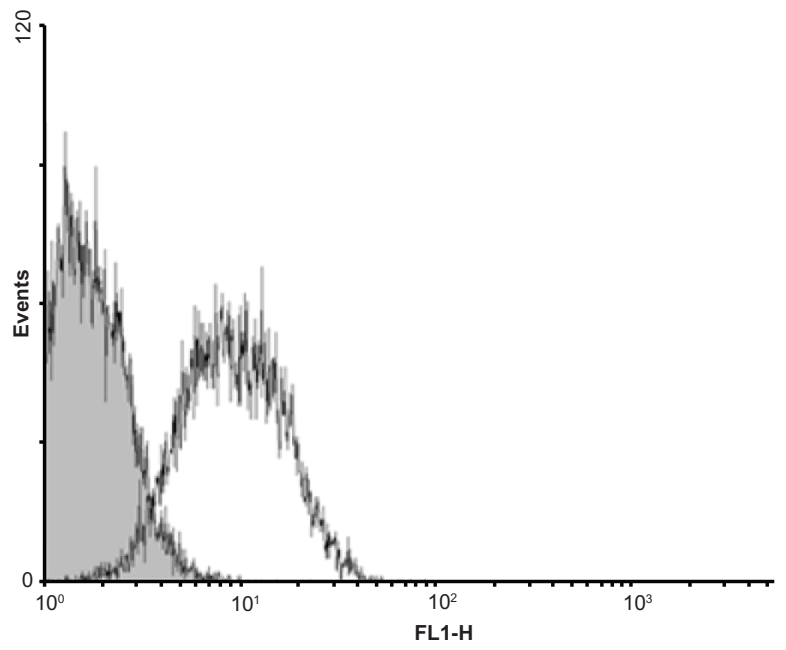

\section{Hyperthermia}

To show that the aptamer-modified NPs can selectively destroy HER2-expressing cells, we used the SK-BR3 cell line overexpressing high levels of the HER2 receptor, and U-87 MG cells as a control. First, we performed the hyperthermia experiment using dextran-coated NPs without a targeting agent. We optimized the concentration of the NPs, as well as their exposure time to the magnetic field and the magnetic field frequency.

In order to eliminate the possible effects of both the magnetic field and the NPs on the cells, the appropriate controls were run. The sample marked as $\mathrm{CN}$ - was treated with the NPs but without the magnetic field, the sample identified as $\mathrm{C}+$ underwent the magnetic field but without the NPs, and the C-sample included the cells that were not subject to either

\section{B}

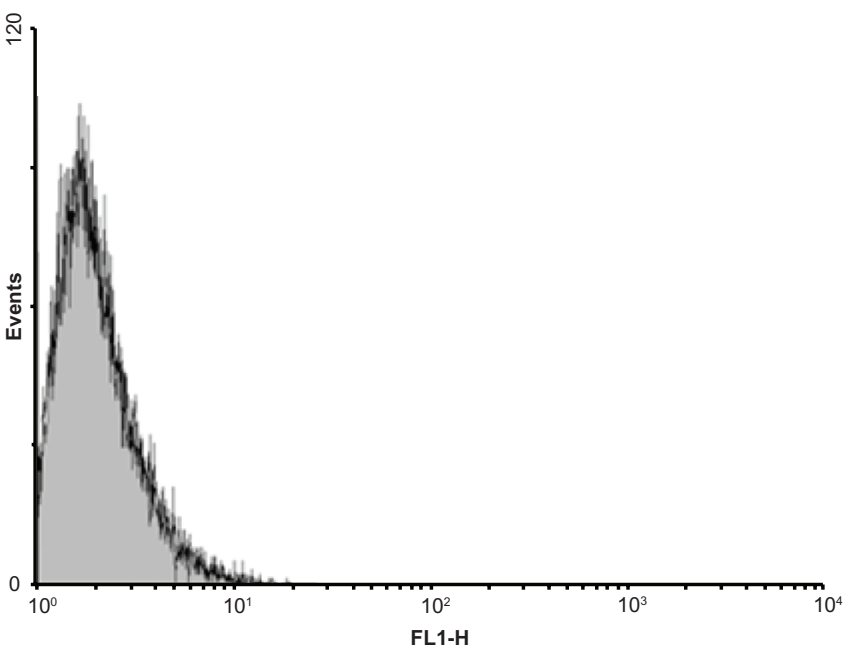

Figure 3 Cell specificity of nanoparticles tagged with an anti-HER2 aptamer.

Notes: (A) SK-BR3 and (B) U-87 MG cell lines were incubated with nanoparticles conjugated with an anti-HER2 aptamer and fluorescein isothiocyanate, and they were then subjected to fluorescence-activated cell sorting analysis. The grey area corresponds to cells without nanoparticles. Abbreviation: HER2, human epidermal growth factor receptor 2. 

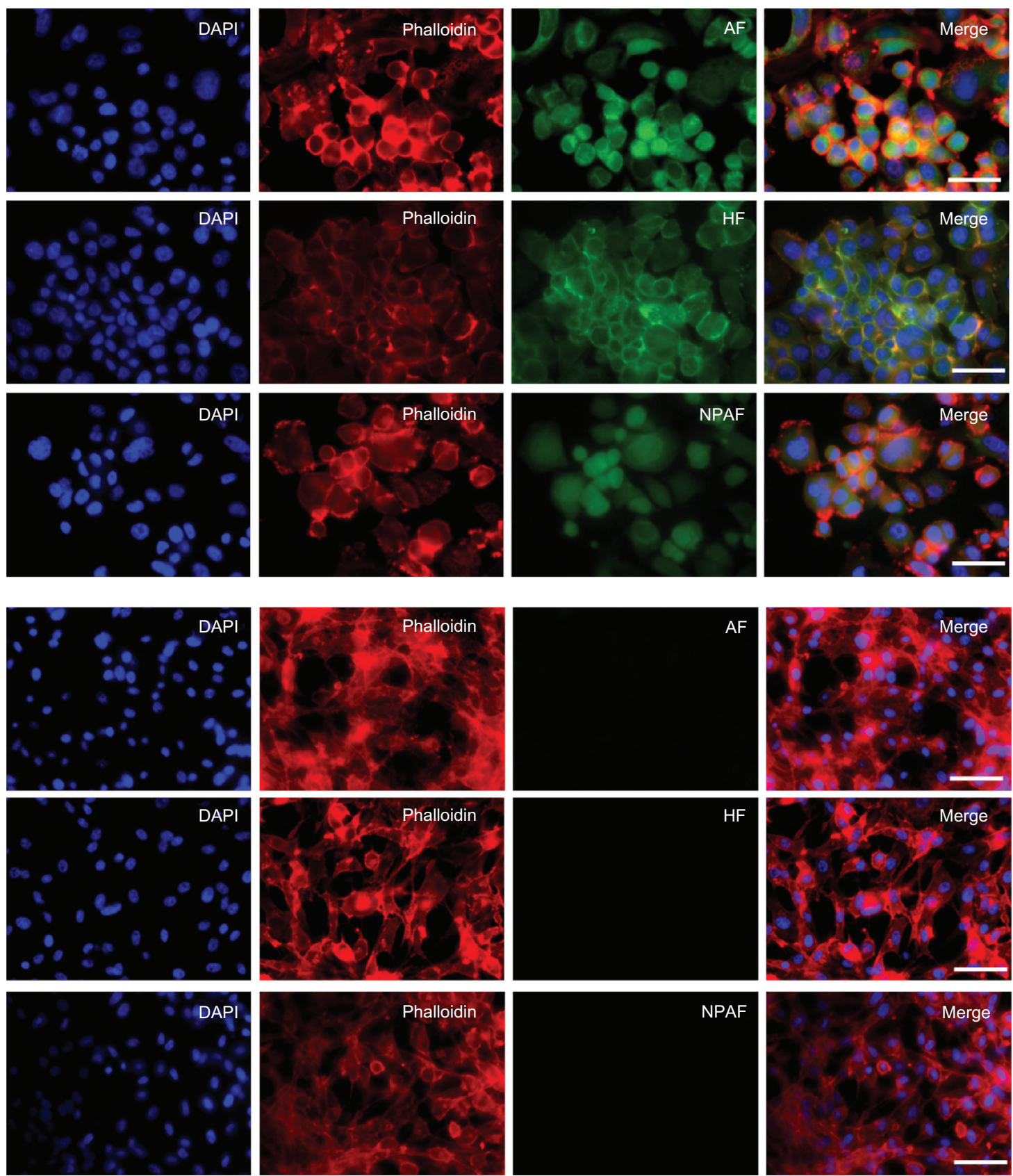

Figure 4 Cell type-specific targeting of anti-HER2 aptamer-tagged nanoparticles.

Notes: SK-BR3 (top three rows) and U-87 MG (bottom three rows) cells were incubated with AF (top), HF (middle), or NPAF (bottom), as examined by fluorescence microscopy. Columns on the left show DAPI staining of nuclei, phalloidin staining of actin, fluorescein signal, and a merged picture. The white bar corresponds to $50 \mu \mathrm{m}$. Abbreviations: DAPI, 4',6-diamidino-2-phenylindole; AF, fluorescein-labeled anti-HER2 aptamer; HF, herceptin; NPAF, nanoparticle-aptamer-fluorescein; HER2, human epidermal growth factor receptor 2 .

the magnetic field or to the NPs. After the hyperthermia experiment, the cells treated with NPs together with magnetic field $(\mathrm{CN}+)$ were compared with all controls.

The results showed that a high concentration of particles $(1 \mathrm{mg} / \mathrm{mL})$ was required for the efficient diminution of cell viability. With the optimized parameters of the magnetic field (300 A, $280 \mathrm{kHz}$ ), up to $50 \%$ of cells could be destroyed (Figure 5). The viability of all the controls $(\mathrm{CN}-, \mathrm{C}+$, and
$\mathrm{C}-$ ) fluctuated around $100 \%$, meaning that neither the magnetic field nor the NPs (separately) were detrimental to the cells. The results were similar for both the SK-BR3 and U-87 MG cell lines. The experiment conducted with non-targeted NPs showed that a relatively high concentration of NPs was required to achieve an $\sim 50 \%$ reduction of the cell viability.

We expected that in using cell-specific targeting of the NPs by means of a receptor-directed aptamer, we could 

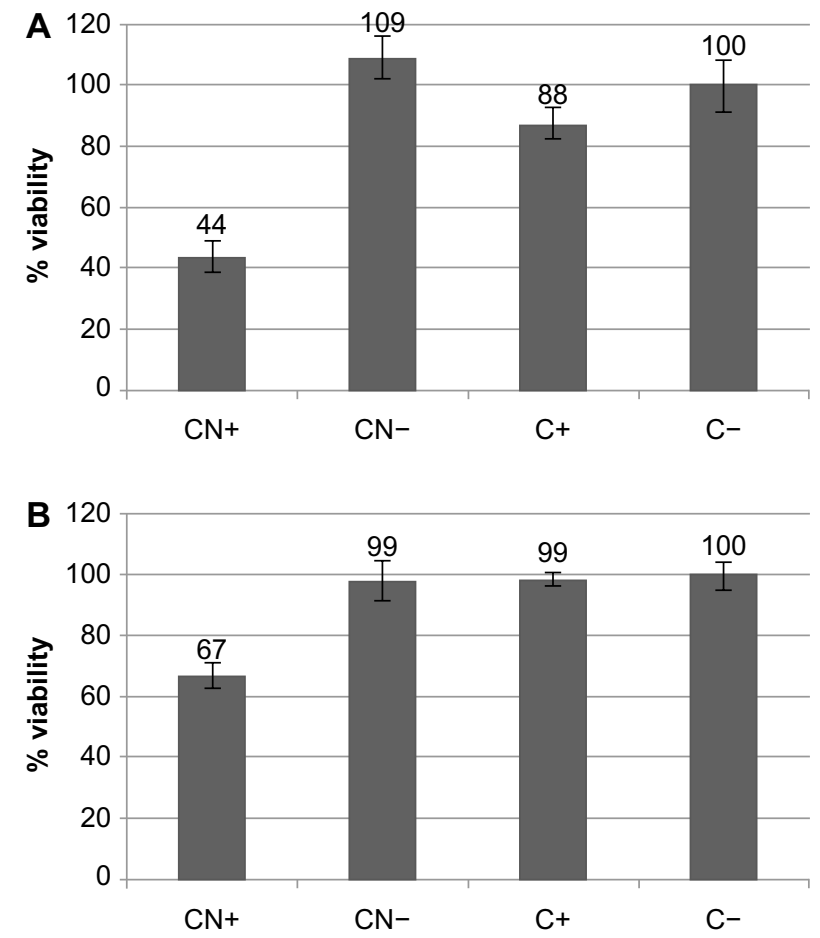

Figure 5 Hyperthermia induced by nanoparticles coated with dextran, without the targeting agent, performed on the SK-BR3 cell line and the U-87 MG cell line.

Notes: (A) SK-BR3 cell line; (B) U-87 MG cell line. The concentration of nanoparticles was I mg/mL.

Abbreviations: $\mathrm{CN}+$, cells treated with nanoparticles and a magnetic field; $\mathrm{CN}-$, cells with nanoparticles without a magnetic field; $\mathrm{C}+$, cells exposed to a magnetic field without nanoparticles; C-, control cells subjected to neither the nanoparticles nor the magnetic field.

achieve greater efficiency in the hyperthermia procedure since the NPs should be concentrated at the cell surface. Experiments similar to those described in the Materials and methods section were conducted with anti-HER2 aptamer-tagged NPs. As shown in Figure 6, the dose of the aptamer-tagged NPs required to produce an $\sim 60 \%$ decrease of the viability of the SK-BR3 cells was only $\sim 11 \mu \mathrm{g} / \mathrm{mL}$, which is 90 -fold less than the dose of non-targeted NPs, other parameters of the experiment being exactly the same. Moreover, the effect was specific to the HER2-overexpressing SK-BR3 cells, since the U-87 MG cells were hardly affected at that dose of targeted NPs (Figure 6). Appropriate controls showed that the death of SK-BR3 cells was indeed caused by the hyperthermic effect, as no other combination of treatments affected their viability.

\section{Discussion}

Recently, magnetic NPs have become a multifunctional tool used in medical practice to increase the sensitivity of diagnostics by enhancing the signal in magnetic resonance imaging, and they are also used to develop new therapeutic strategies that employ the phenomenon of hyperthermia or targeted
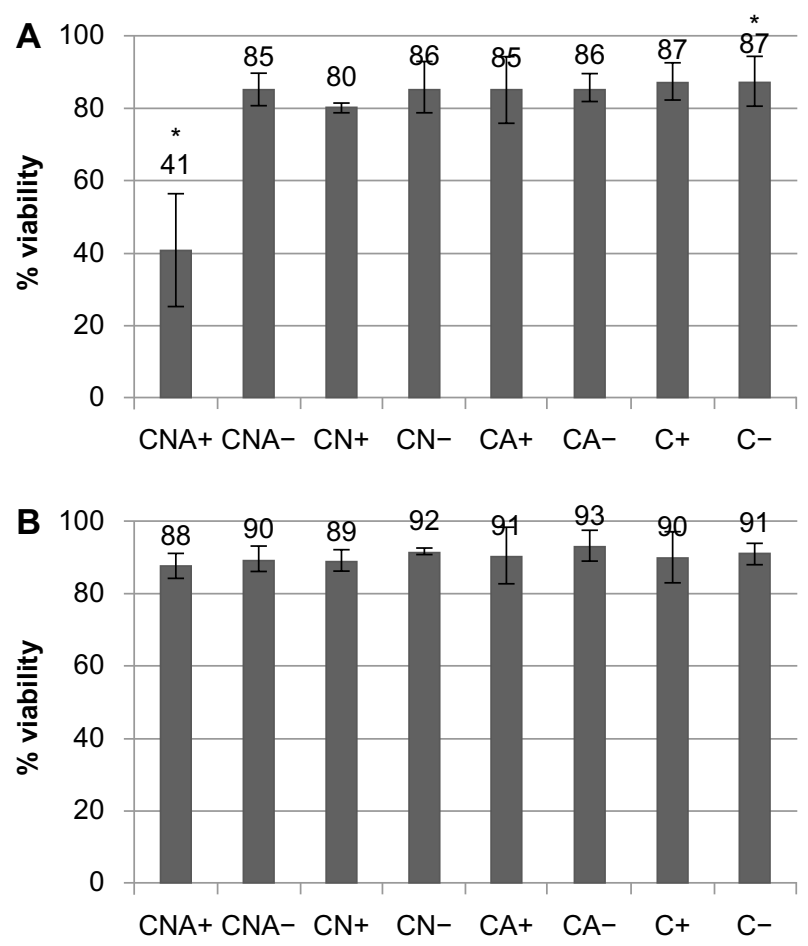

Figure 6 Hyperthermia induced by aptamer-tagged nanoparticles performed on the SK-BR3 cell line (top) and U-87 MG cell line (bottom).

Notes: (A) SK-BR3 cell line; (B) U-87 MG cell line. The concentration of nanoparticles was $I$ I $\mu \mathrm{g} / \mathrm{mL}$. *Statistically significant difference between the CNA+ and $C$ - samples, $P<0.05$.

Abbreviations: CNA+, cells treated with aptamer-modified nanoparticles and a magnetic field; CNA-, cells treated with aptamer modified nanoparticles without a magnetic field; $\mathrm{CN}+$, cells treated with nanoparticles and a magnetic field; $\mathrm{CN}-$, cells with nanoparticles without a magnetic field; $\mathrm{CA}+$, cells treated with aptamer and a magnetic field; CA-, cells treated with aptamer without a magnetic field; $\mathrm{C}+$, cells exposed to a magnetic field without nanoparticles; C-, control cells subjected to neither nanoparticles nor to a magnetic field.

drug delivery. Local hyperthermia, supported by magnetic NPs conjugated with targeting agents, assists chemo- or radiotherapy. Magnetic hyperthermia decreases the required dose of chemotherapeutics, reducing the side effects of chemotherapy; ${ }^{18}$ in this way, this approach is particularly efficient in cancers that are resistant to chemotherapeutics. ${ }^{19}$

Aptamers are becoming valuable molecular tools in diagnostics and therapeutics, and they are used as bioconjugates in combination with drugs and nanomaterials in biosensors, ${ }^{20}$ bioseparation, targeted drug delivery, ${ }^{21}$ and cancer treatment. ${ }^{22,23}$

In this paper, we used dextran-coated ferric oxide NPs tagged with an aptamer directed against a cell surface receptor to show the ability of such a system to selectively recognize receptor-presenting cells. In conjunction with superparamagnetic properties, the construct was designed to improve the specificity of the hyperthermic effect. The HER2 receptor is frequently overexpressed in breast cancer and it is one of the most popular targets in cancer therapies. 
According to our knowledge, this is the first study of an HER2-specific aptamer combined with magnetic NPs for application in targeted hyperthermia.

Hadjipanayis et al ${ }^{24}$ constructed NPs that were modified with an anti-epidermal growth factor receptor (EGFR) antibody, and the authors showed their applicability in glioblastoma treatment. Magnetic resonance imaging showed that NPs accumulated in tumor tissue, which indicated that they could be used for specific drug delivery and hyperthermia.

Hyperthermia provided by AuNPs was evaluated by Raoof et al. ${ }^{25}$ The particles were conjugated with an antibody against EGFR via an $\mathrm{Au}-\mathrm{S}$ interaction. The bioconjugate was internalized by EGFR-overexpressing cells, which were then subjected to radiofrequency. The AuNPs exhibited the hyperthermic effect under the radio frequency field and caused cell death. A specific linker forming the $\mathrm{Au}-\mathrm{S}$ bond had to be used to conjugate the antibody with the AuNPs.

Specific linkers are commonly used for the conjugation of proteins with other molecules or particles. Disadvantages of that method are connected with the yield of the reaction and the possibility of the linkers binding to undesired functional groups. Nonspecific modifications covering the active sites are possible reasons for the decreased activity. No such problems exist when synthetic aptamers are used.

In this paper, we showed that aptamer-modified superparamagnetic NPs are functional and selective. They were effective in the hyperthermic killing of cancer cells that overexpressed an appropriate receptor at a dosage that was approximately 100 -fold lower when compared to that of non-targeted NPs. The possibility of using a low concentration of the NPs is highly desirable in light of the side effects associated with treatment.

Magnetic NPs administered intravenously are cleared from the bloodstream by the reticuloendothelial system. For the magnetic contrast agents administered in clinical use (ferumoxtran-10; Feridex IV ${ }^{\circledR}$, Berlex, Inc, Wayne, NJ, USA), the time of clearance varies from 1 hour to 36 hours, and it depends mainly on the size and type of the coating agent. The elimination of magnetic fluid is carried out by the liver, spleen, and bone marrow. Moreover, metabolism efficiency varies as a function of the injected dose and the biodistribution, ${ }^{26}$ so a lower effective concentration is likely to cause milder side effects.

At the tissue level, the immature tumor vasculature is porous and leaky, so untargeted NPs are rapidly flushed away; therefore, the process of heating is not efficient. Targeted NPs bound to receptors on the surface of cancer cells offer high effective local concentrations..$^{27,28}$
To summarize, targeted NPs combined with hyperthermia efficiently cause cell death in vitro. Application of such NPs in therapy may decrease the side effects caused by the indiscriminate killing of nontumor cells, as well as any damage to the liver, spleen, or bone marrow.

\section{Acknowledgments}

The research was supported by the Wroclaw Research Centre EIT+ under the project, "Biotechnologies and advanced medical technologies" - BioMed (POIG.01.01.02-02003/08), which was financed from the European Regional Development Fund (Operational Programme Innovative Economy, 1.1.2).

We thank Professor Eugeniusz Zych and Professor Tadeusz Lis from the Faculty of Chemistry, University of Wroclaw for their help with the TEM and XRD analysis. We are also grateful to Roman Gorzelniak, MSc, for performing SQUID measurement.

\section{Disclosure}

The authors report no conflicts of interest in this work.

\section{References}

1. Parak WJ, Gerion D, Pellegrino T, et al. Biological applications of colloidal nanocrystals. Nanotechnology. 2003;14:R15.

2. Pankhurst QA, Connolly J, Jones SK, Dobson J. Applications of magnetic nanoparticles in biomedicine. J Phys D Appl Phys. 2003; 36:R167.

3. Murray CB, Kagan CR, Bawendi MG. Synthesis and characterization of monodisperse nanocrystals and close-packed nanocrystal assemblies. Annual Review of Materials Science. 2000;30:545-610.

4. Farokhzad OC, Langer R. Impact of nanotechnology on drug delivery. ACS Nano. 2009;3(1):16-20.

5. Jun YW, Seo JW, Cheon J. Nanoscaling laws of magnetic nanoparticles and their applicabilities in biomedical sciences. Acc Chem Res. 2008;41(2):179-189.

6. Jordan A, Scholz R, Wust P, Fähling H, Felix R. Magnetic fluid hyperthermia $(\mathrm{MFH})$ : cancer treatment with $\mathrm{AC}$ magnetic field induced excitation of biocompatible superparamagnetic nanoparticles. J Magn Magn Mater. 1999;201(1-3):413-419.

7. Jordan A, Scholz R, Maier-Hauff K, et al. Presentation of a new magnetic field therapy system for the treatment of human solid tumors with magnetic fluid hyperthermia. J Magn Magn Mater. 2001; 225(1-2):118-126.

8. Hildebrandt B, Wust $\mathrm{P}, \mathrm{Ahlers} \mathrm{O}$, et al. The cellular and molecular basis of hyperthermia. Crit Rev Oncol Hematol. 2002;43(1):33-56.

9. Wust P, Hildebrandt B, Sreenivasa G, et al. Hyperthermia in combined treatment of cancer. Lancet Oncol. 2002;3(8):487-497.

10. van der Zee J, van Rhoon GC. Hyperthermia is effective in improving clinical radiotherapy results. Int J Radiat Oncol Biol Phys. 2006;66(2): 633-634; author reply 634.

11. Huang YF, Chang HT, Tan W. Cancer cell targeting using multiple aptamers conjugated on nanorods. Anal Chem. 2008;80(3):567-572.

12. Yang L, Zhang X, Ye M, et al. Aptamer-conjugated nanomaterials and their applications. Adv Drug Deliv Rev. 2011;63(14-15):1361-1370.

13. Huang CC, Huang YF, Cao Z, Tan W, Chang HT. Aptamer-modified gold nanoparticles for colorimetric determination of platelet-derived growth factors and their receptors. Anal Chem. 2005;77(17):5735-5741. 
14. Yigit MV, Mazumdar D, Lu Y. MRI detection of thrombin with aptamer functionalized superparamagnetic iron oxide nanoparticles. Bioconjug Chem. 2008;19(2):412-417.

15. Cao Z, Tong R, Mishra A, et al. Reversible cell-specific drug delivery with aptamer-functionalized liposomes. Angew Chem Int Ed Engl. 2009;48(35):6494-6498.

16. Löfås S, Johnsson B. A novel hydrogel matrix on gold surfaces in surface plasmon resonance sensors for fast and efficient covalent immobilization of ligands. J Chem Soc Chem Commun. 1990:1526-1528.

17. Hradil J, Pisarev A, Babic M, Horak D. Dextran-modified iron oxide nanoparticles. China Particuology. 2007;5:162-168.

18. Silva AC, Oliveira TR, Mamani JB, et al. Application of hyperthermia induced by superparamagnetic iron oxide nanoparticles in glioma treatment. Int J Nanomedicine. 2011;6:591-603.

19. Aljarrah K, Mhaidat NM, Al-Akhras MA, et al. Magnetic nanoparticles sensitize MCF-7 breast cancer cells to doxorubicin-induced apoptosis. World J Surg Oncol. 2012;10:62.

20. Lu W, Arumugam SR, Senapati D, et al. Multifunctional oval-shaped gold-nanoparticle-based selective detection of breast cancer cells using simple colorimetric and highly sensitive two-photon scattering assay. ACS Nano. 2010;4(3):1739-1749.

21. Hernandez FJ, Hernandez LI, Pinto A, Schäfer T, Özalp VC. Targeting cancer cells with controlled release nanocapsules based on a single aptamer. Chem Commun (Camb). 2013;49(13):1285-1287.
22. Cohen BA, Bergkvist M. Targeted in vitro photodynamic therapy via aptamer-labeled, porphyrin-loaded virus capsids. J Photochem Photobiol B. 2013;121:67-74.

23. Lee YJ, Kim IS, Park SA, et al. Periostin-binding DNA aptamer inhibits breast cancer growth and metastasis. Mol Ther. 2013;21(5):1004-1013.

24. Hadjipanayis CG, Machaidze R, Kaluzova M, et al. EGFRvIII antibody-conjugated iron oxide nanoparticles for magnetic resonance imaging-guided convection-enhanced delivery and targeted therapy of glioblastoma. Cancer Res. 2010;70(15):6303-6312.

25. Raoof M, Corr SJ, Kaluarachchi WD, et al. Stability of antibodyconjugated gold nanoparticles in the endolysosomal nanoenvironment: implications for noninvasive radiofrequency-based cancer therapy. Nanomedicine. 2012;8(7):1096-1105.

26. Corot C, Robert P, Idée JM, Port M. Recent advances in iron oxide nanocrystal technology for medical imaging. Adv Drug Deliv Rev. 2006;58(14):1471-1504.

27. Diagaradjane P, Orenstein-Cardona JM, Colón-Casasnovas NE, et al. Imaging epidermal growth factor receptor expression in vivo: pharmacokinetic and biodistribution characterization of a bioconjugated quantum dot nanoprobe. Clin Cancer Res. 2008;14(3):731-741.

28. Diagaradjane P, Shetty A, Wang JC, et al. Modulation of in vivo tumor radiation response via gold nanoshell-mediated vascular-focused hyperthermia: characterizing an integrated antihypoxic and localized vascular disrupting targeting strategy. Nano Lett. 2008;8(5):1492-1500. 


\section{Supplementary materials}
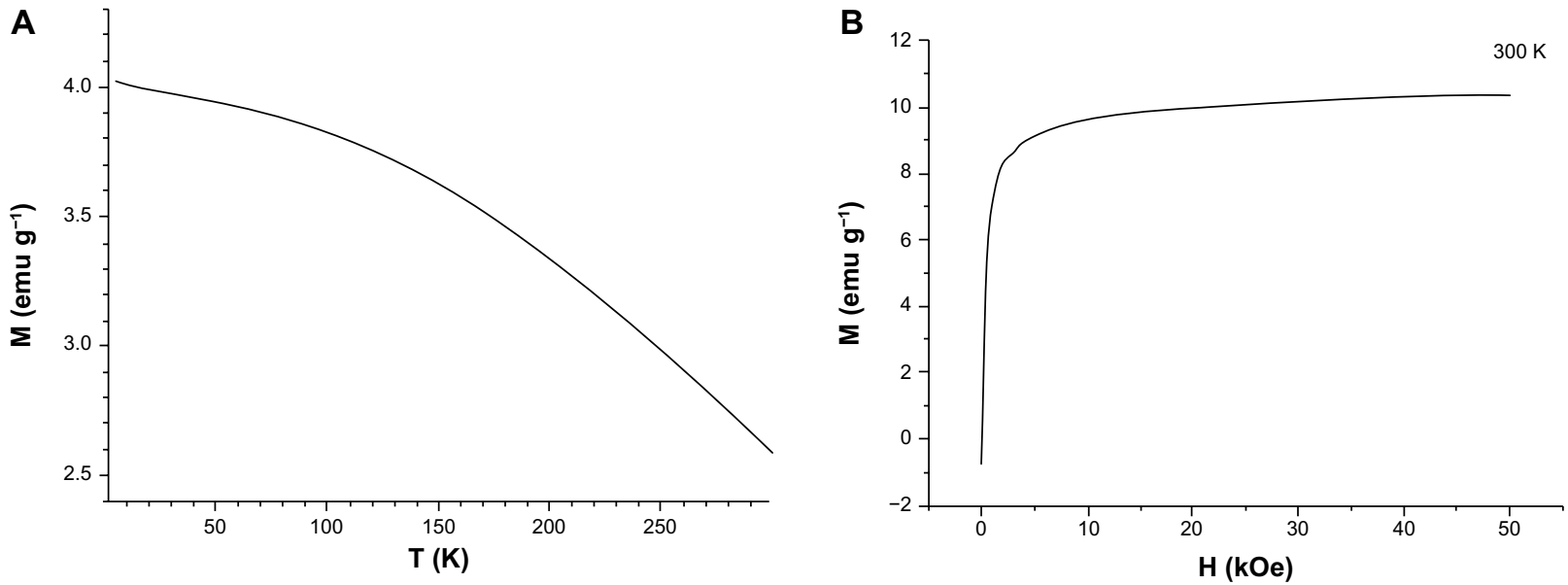

Figure SI Superconducting quantum interference device magnetometry measurement of magnetization of the iron oxide nanoparticles. Notes: (A) Temperature dependence of sample magnetization. (B) Half of the hysteresis loop.

Abbreviations: $\mathrm{M}$, magnetization; $\mathrm{H}$, magnetic field; K, Kelvin; kOe, kilo Oersted.

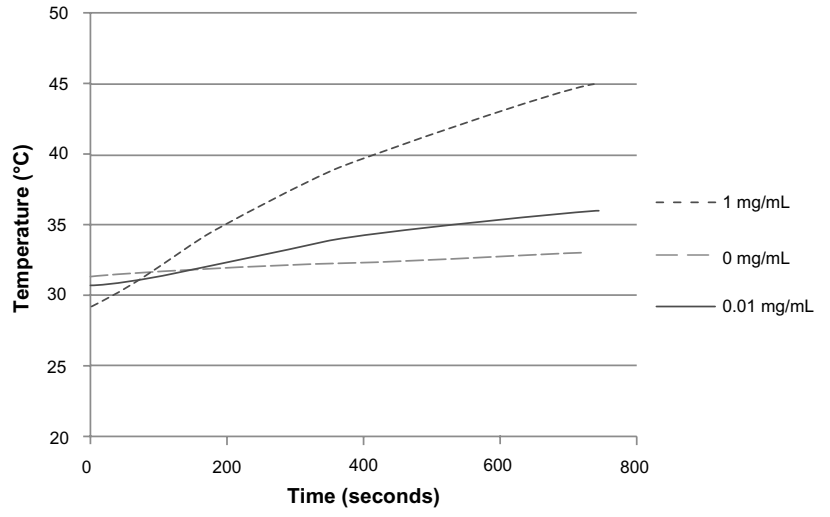

Figure S2 The rise of the temperature of magnetic nanoparticles coated with dextran and subject to a magnetic field ( $300 \mathrm{~A}, 280 \mathrm{kHz})$.

International Journal of Nanomedicine

\section{Publish your work in this journal}

The International Journal of Nanomedicine is an international, peerreviewed journal focusing on the application of nanotechnology in diagnostics, therapeutics, and drug delivery systems throughout the biomedical field. This journal is indexed on PubMed Central, MedLine, CAS, SciSearch $\AA$, Current Contents $\AA /$ Clinical Medicine,
Journal Citation Reports/Science Edition, EMBase, Scopus and the Elsevier Bibliographic databases. The manuscript management system is completely online and includes a very quick and fair peer-review system, which is all easy to use. Visit http://www.dovepress.com/ testimonials.php to read real quotes from published authors. 